\title{
Thermopower of a superconducting single-electron transistor
}

\author{
Marko Turek, ${ }^{1, *}$ Jens Siewert, ${ }^{1,2}$ and Klaus Richter ${ }^{1}$ \\ ${ }^{1}$ Institut für Theoretische Physik, Universität Regensburg, D-93040 Regensburg, Germany \\ ${ }^{2}$ Dipartimento di Metodologie Fisiche e Chimiche per l'Ingegneria, Universita di Catania, I-95125 Catania, Italy
}

(Received 10 March 2005; published 6 June 2005)

\begin{abstract}
We present a linear-response theory for the thermopower of a single-electron transistor consisting of a superconducting island weakly coupled to two normal-conducting leads. The thermopower shows oscillations with the same periodicity as the conductance and is rather sensitive to the size of the superconducting gap $\Delta$. In particular, the previously studied sawtooth-like shape of the thermopower for a normal-conducting singleelectron device is qualitatively changed even for small gap energies.
\end{abstract}

DOI: 10.1103/PhysRevB.71.220503

PACS number(s): 72.15.Jf, 73.23.Hk, 74.45.+c

The transport properties of small conducting grains in the Coulomb blockade (CB) regime have extensively been studied during the past years. This regime is characterized by a unique energy scale, the so-called charging energy $E_{c}$ of the grain (see below). The most prominent phenomenon is the occurence of CB oscillations in the low-temperature conductance of a small grain weakly coupled to the leads. ${ }^{1}$ Recently, thermoelectric effects in single-electron devices such as the thermopower have attracted growing interest. ${ }^{2-11}$ The thermopower is related to the current that arises due to a finite temperature difference between the two leads. ${ }^{14}$ It yields additional information about the kinetics of the system as it measures the average energy of the electrons carrying the current through the system. Therefore, some type of electronhole asymmetry in the system is necessary in order to observe a nonvanishing thermopower.

In analogy to the $\mathrm{CB}$ oscillations of the conductance, the thermopower of a small grain shows oscillations of the same periodicity but with sawtooth-like shape. ${ }^{2,3}$ In contrast to the conductance this dependence on the external gate voltage is very sensitive to the conditions under which the thermoelectric transport occurs. This sensitivity has been demonstrated, e.g., for the transition from the sequential tunneling regime to the cotunneling regime. ${ }^{4,5}$ Recently, the thermopower of open quantum dots with strong coupling to the leads was investigated. ${ }^{6-8}$ Further, the influence of Kondo correlations in ultrasmall quantum dots on the thermoelectric effects was studied in Refs. 9 and 10, while the thermopower of a molecule with internal degrees of freedom and weakly coupled to the leads was discussed in Ref. 11.

It is surprising that, despite the enormous interest in superconducting single-electron transistors (SETs), the thermopower of such structures has not been investigated yet. In this work we study theoretically the thermopower of a normal-superconducting-normal SET (NSN SET), i.e., a small superconducting island that is weakly coupled to normal-conducting leads (cf. Fig. 1), in an experimentally accessible regime. We show that even for rather small superconducting gaps (compared to the charging energy of the island) the functional dependence of the thermopower on the gate voltage is qualitatively changed while its amplitude remains on the same order of magnitude. This is in clear contrast to the corresponding results for the conductance where the most pronounced effect is a suppression of the amplitude with increasing gap size. ${ }^{12}$
Thermopower of single-electron devices. In the following we investigate the regime of single-electron tunneling through a superconducting island with a charging energy $E_{c}$ that is large compared to the temperature $T$, i.e., $E_{c} \equiv e^{2} /(2 C) \gg T$, where $-e$ is the electron charge and $C$ the capacitance of the island. The temperature is assumed to be larger than the crossover temperature for parity effects. ${ }^{13}$ The electronic spectrum of the grain in the normal-conducting state is assumed to be continuous and the conductances of the tunnel barriers are much smaller than $e^{2} / h$. This implies that cotunneling processes can be neglected and sequential tunneling dominates. Taking into account the external electrostatic potential $\phi \propto V_{g}$ imposed by the gate voltage $V_{g}$, the total electrostatic energy of the island can be expressed as

$$
E_{n}(\phi)=E_{c}\left(n^{2}-2 n \frac{C}{e} \phi\right),
$$

where $n$ is the number of excess electrons on the island. To add one electron to the island an energy $u_{n}(\phi)$

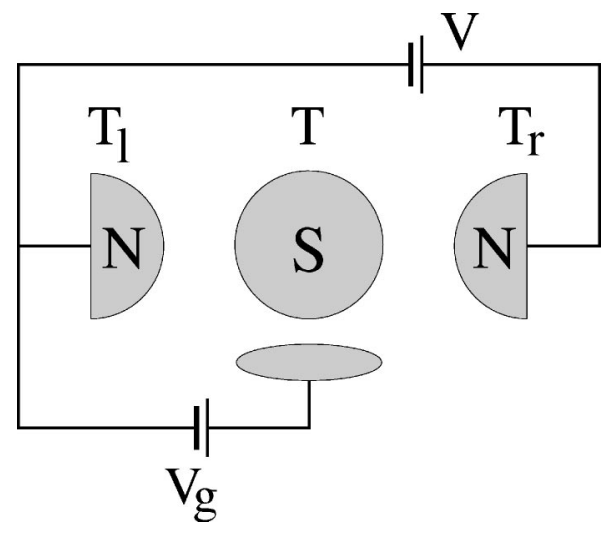

FIG. 1. The NSN SET consists of a superconducting island $(S)$ which is coupled to two normal-conducting leads $(N)$ via tunnel barriers. The electrostatic potential of the island can be controlled by the gate voltage $V_{g}$. The current through the system is due to the bias voltage $V$ or a temperature difference $\Delta T=T_{l}-T_{r}$ between the two leads. To measure the thermopower $S=-V / \Delta T$ as a function of the gate voltage $V_{g}$ the bias $V$ is adjusted in such a way that the corresponding current exactly cancels the current due to the temperature difference. 
$\equiv E_{n+1}(\phi)-E_{n}(\phi)$ is required. At low temperatures $T \ll E_{c}$ the electronic transport is Coulomb blocked. A current flows only at potentials $\phi \approx \phi_{n}$ where $\phi_{n}$ is given by the condition $u_{n}\left(\phi_{n}\right)=0$. With Eq. (1) one finds

$$
\phi_{n}=\frac{e}{C}\left(n+\frac{1}{2}\right)
$$

The current through the device can be either due to a transport voltage $V$ or a temperature difference $\Delta T=T_{l}-T_{r}$ between the two leads (see Fig. 1). Throughout this work we consider the linear-response regime, i.e., $e V / E_{c} \ll 1$ and $\Delta T / T \ll 1$.

For zero-temperature difference the linear response to the voltage $V$ is given by the conductance $G_{V}$. The conductance as a function of the potential $\phi$ it shows that the well-known CB peaks at $\phi=\phi_{n}$. In the general case with $\Delta T \neq 0$ the current

$I=G_{V} V+G_{T} \Delta T$. The thermopower of the system is defined by the ratio of voltage and temperature difference for vanishing current, i.e.,

$$
S \equiv-\left.\lim _{\Delta T \rightarrow 0} \frac{V}{\Delta T}\right|_{I=0}=\frac{G_{T}}{G_{V}} .
$$

Following Matveev ${ }^{15}$ a rather intuitive interpretation of the $\mathrm{CB}$ oscillations of the thermopower can be given in terms of the average energy $\langle\xi\rangle$ of the electrons that carry the current through the system

$$
S=-\frac{\langle\xi\rangle}{e T}
$$

Here, we briefly review the argument in the NNN case (normal-conducting island) considering a two-state approximation (valid for $T \ll E_{c}$ ). If in equilibrium there are $n$ electrons on the grain two different transport cycles are possible: one can first add an electron and then remove it again $[n \rightarrow(n+1) \rightarrow n]$ or vice versa $[n \rightarrow(n-1) \rightarrow n]$. In the first case the average energy is given by the difference of charging energies, $\langle\xi\rangle=\left(E_{n+1}-E_{n}\right) / 2$, while in the second case it is $\langle\xi\rangle=\left(E_{n}-E_{n-1}\right) / 2$. The potential $\phi$ determines which of the processes is more likely to occur. For example, at $\phi \gtrsim \phi_{n}$ the probability of having $n$ or $n+1$ electrons in the grain is higher than the probability for $n-1$ electrons. Thus, the first process dominates, and according to Eq. (4) one finds the well-known sawtooth behavior

$$
S_{\mathrm{NNN}}(\phi)=-\frac{u_{n}(\phi)}{2 e T} \quad \text { for } C\left|\phi-\phi_{n}\right| / e<\frac{1}{2}
$$

(see inset of Fig. 2). The extrema of the sawtooth at $e\left|\phi-\phi_{n}\right| \approx E_{c}$ are rounded due to the finite temperature.

Thermopower of NSN SET. In the following we consider the case of a superconducting grain with a gap $\Delta<E_{c}$. In a stationary state the currents through the left and the right tunnel junction are equal, i.e., $I=I_{l}=I_{r}$. In order to evaluate the thermopower we make use of relation (3) and calculate the linear response of the current to a voltage $V$ or a temperature difference $\Delta T=T_{l}-T_{r}$. According to the "orthodox theory" the current through the system can be written as

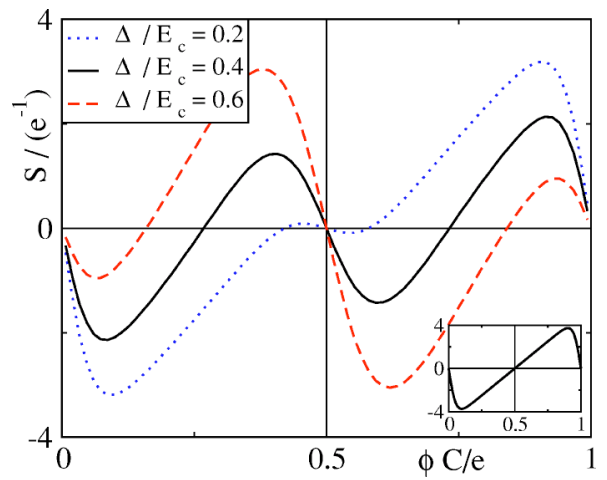

FIG. 2. Thermopower $S(\phi)$ of the NSN setup with $\Delta<E_{c}$ for different values of the superconducting gap $\Delta$ at a temperature $T / E_{c}=0.1$. The inset shows the corresponding result for the NNN SET. Note that the conductance peak would be located at $\phi=\phi_{0} \equiv 0.5 e / C$ [cf. Eq. (2)].

$$
I=-e \sum_{n} P_{n}\left[\Gamma_{r}^{n \rightarrow n-1}-\Gamma_{r}^{n \rightarrow n+1}\right],
$$

where $P_{n}$ is the stationary probability for finding $n$ electrons on the island, $\Gamma_{r}^{n \rightarrow n-1}$ is the tunneling rate of an electron from the island to the right lead, and $\Gamma_{r}^{n \rightarrow n+1}$ denotes the tunneling rate from the right lead to the island. The rates $\Gamma_{r}$, and correspondingly $\Gamma_{l}$, can be written in terms of the Fermi function $f(x) \equiv 1 /[1+\exp (x)]$ as

$$
\begin{aligned}
\Gamma_{r, l}^{n+1 \rightarrow n}= & 2 \frac{G_{r, l}}{e^{2}} \int_{\Delta}^{\infty} d E \frac{E}{\sqrt{E^{2}-\Delta^{2}}}\left[f\left(-\frac{E \pm e V / 2+u_{n}}{T_{r, l}}\right) f\left(\frac{E}{T}\right)\right. \\
& \left.+f\left(\frac{E \mp e V / 2-u_{n}}{T_{r, l}}\right) f\left(-\frac{E}{T}\right)\right] \\
\Gamma_{r, l}^{n \rightarrow n+1}= & 2 \frac{G_{r, l}}{e^{2}} \int_{\Delta}^{\infty} d E \frac{E}{\sqrt{E^{2}-\Delta^{2}}}\left[f\left(\frac{E \pm e V / 2+u_{n}}{T_{r, l}}\right) f\left(-\frac{E}{T}\right)\right. \\
& \left.+f\left(\frac{-E \pm e V / 2+u_{n}}{T_{r, l}}\right) f\left(\frac{E}{T}\right)\right] .
\end{aligned}
$$

Here, $E$ is the energy of the quasiparticles in the superconductor and $G_{r, l}$ is the conductance of the right and left tunnel junctions, respectively (see, e.g., Ref. 14). The first term in Eq. (7) corresponds to the annihilation of a quasiparticle while the second term yields the contribution due to the creation of a quasiparticle.

The probabilities $P_{n}$ in Eq. (6) can be obtained from the stationary solution of a kinetic equation. They obey the relation $^{2}$

$$
P_{n+1}=\frac{\Gamma_{l}^{n \rightarrow n+1}+\Gamma_{r}^{n \rightarrow n+1}}{\Gamma_{l}^{n+1 \rightarrow n}+\Gamma_{r}^{n+1 \rightarrow n}} P_{n},
$$

with $\sum_{n} P_{n}=1$. The set of Eqs. (6)-(9) allows us to calculate the transport coefficients $G_{V}$ and $G_{T}$. Together with relation (3) the thermopower $S$ can be obtained as a function of the potential $\phi$ for different parameters $\Delta$ and $T$. The results of our calculation are shown in Fig. 2. Compared to the sawtooth-like behavior of the thermopower in the NNN case 
(see inset of Fig. 2) the shape is significantly changed for gaps $\Delta$ smaller than the charging energy $E_{c}$. For increasing $\Delta \rightarrow E_{c}$ the extrema of $S(\phi)$ move clearly away from $\phi_{n} \pm e /(2 C)$.

We emphasize that the qualitative change of the thermopower as a function of the potential $\phi$ due to a finite gap is much more pronounced than the changes in the conductance where one merely finds a broadening of the Coulombblockade peaks together with an overall exponential suppression of the current. Note, however, that the order of magnitude of the thermopower remains the same independently of the gap size. This behavior can be understood by analyzing Eqs. (6)-(9) in the low-temperature regime where the major contribution to the electronic transport is due to only two charge states.

Two-state approximation. For low temperatures $T \ll E_{c}$ there are at most two probabilities that assume a finite value for a given $\phi$, e.g., $P_{0}$ and $P_{1}$ for $C\left|\phi-\phi_{0}\right| / e<1 / 2$. All remaining probabilities are exponentially small in the parameter $E_{c} / T$. To first order in the perturbations $V$ and $\Delta T$ the current (6) can be expressed in terms of the unperturbed probabilities $P_{0,1}^{(0)}$ and the exact rates $\Gamma_{l, r}$, Eqs. (7) and (8), as

$$
\begin{aligned}
I= & \frac{e}{G_{l}+G_{r}}\left[P_{0}^{(0)}\left(G_{l} \Gamma_{r}^{0 \rightarrow 1}-G_{r} \Gamma_{l}^{0 \rightarrow 1}\right)\right. \\
& \left.+P_{1}^{(0)}\left(G_{r} \Gamma_{l}^{1 \rightarrow 0}-G_{l} \Gamma_{r}^{1 \rightarrow 0}\right)\right] .
\end{aligned}
$$

The zeroth-order probabilities can be obtained from Eq. (9) and the condition $P_{0}^{(0)}+P_{1}^{(0)}=1$, which yields $P_{0,1}^{(0)} \approx f\left[\mp u_{0}(\phi) / T\right]$ if exponentially small corrections are neglected.

First, we discuss the thermopower for potentials $\phi$ close to $\phi_{0}$, i.e., $C\left|\phi-\phi_{0}\right| / e<\Delta /\left(2 E_{c}\right)$ where $\left|u_{0}(\phi)\right|<\Delta$. In this range of $\phi$, the changes in the thermopower due to the superconducting properties of the island are most prominent (see Fig. 2). For gap energies that are not too small $\Delta \leq E_{c}$ we can neglect contributions that are exponentially small in $\Delta / T$. Thus we find from Eqs. (7), (8), and (10) together with relation (3) the asymptotic result

$$
S(\phi) \approx-\frac{u_{0}(\phi)}{e T}\left(1-\frac{\tilde{\Delta}(T)}{u_{0}(\phi)} \tanh \left[\frac{u_{0}(\phi)}{2 T}\right]\right) .
$$

Here, $\widetilde{\Delta}(T) \equiv-\Delta K_{1}^{\prime}(\Delta / T) / K_{1}(\Delta / T)$ where $K_{1}^{\prime}(x)$ is the derivative of the Bessel function $K_{1}(x)$. In contrast to the NNN case we find $S=0$ not only for $u_{0}(\phi)=0$ but also at $\left|u_{0}(\phi)\right| \approx \tilde{\Delta}$ (see Fig. 2). Between these two zeros the thermopower reaches its extrema at $\left|u_{\max }\right|$ $\approx 2 T^{-1} \operatorname{arccosh} \sqrt{\widetilde{\Delta} /(2 T)}$. This unique behavior, that the slope of $S(\phi)$ changes its sign at $\phi=\phi_{0}$, occurs even for small gap values $\tilde{\Delta} \sim 2 T$. In the low-temperature limit $\Delta / T \gg 1$ the temperature-dependent "effective gap" $\widetilde{\Delta}(T)$ in Eq. (11) is simply replaced by the constant gap $\Delta$. On the other hand, Eq. (11) also reproduces the limit $\Delta \rightarrow 0$ correctly as it gives $\widetilde{\Delta} \rightarrow T$ leading to the NNN result of Eq. (5).

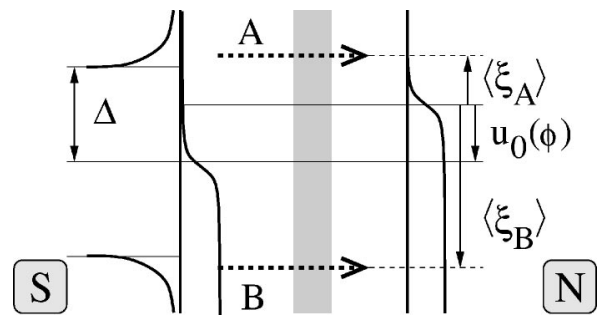

FIG. 3. Tunneling processes from the superconducting island to the right lead for $\left|u_{0}(\phi)\right|<\Delta$ : annihilation of a quasiparticle (A) and creation of a quasiparticle (B). The corresponding average energies (measured from the Fermi energy of the lead) are $\left\langle\xi_{A}\right\rangle>0$ and $\left\langle\xi_{B}\right\rangle<0$. Depending on which of the processes is dominant the sign of $S$ results according to Eq. (4).

Next we consider the thermopower in the $\phi$ range $\Delta<\left|u_{0}(\phi)\right|<E_{c}$, i.e., $\Delta /\left(2 E_{c}\right)<C\left|\phi-\phi_{0}\right| / e<1 / 2$. In this case Eqs. (10) and (3) approximately yield

$$
S(\phi) \approx-\frac{u_{0}(\phi)}{2 e T}\left(1-\left[\frac{\Delta}{u_{0}(\phi)}\right]^{2 \operatorname{arcosh}\left[\frac{\left|u_{0}(\phi)\right|}{\Delta}\right]} \frac{\sqrt[\Delta]{1-\left[\frac{\Delta}{u_{0}(\phi)}\right]^{2}}}{\sqrt{1}}\right) .
$$

From this result we find $S=0$ for $\left|u_{0}(\phi)\right|=\Delta$, which agrees with Eq. (11) in the low-temperature limit where $\widetilde{\Delta} \rightarrow \Delta$. The second term in Eq. (12) depends only weakly on $\phi$ but gives an overall shift by $\Delta / 2$ (see Fig. 2). Therefore, the dependence of $S$ on the potential $\phi$ is almost linear so that the extrema for $u_{0}(\phi) \rightarrow \pm E_{c}$ are approximately given by $S_{\max } \approx \mp\left(E_{c}-\Delta\right) /(2 e T)$, respectively. Similar to the NNN case there is a thermal smoothing close to the edge of the $\phi$ interval where $E_{c}-\left|u_{0}(\phi)\right| \lesssim T$. This is because for these values of $\phi$ charge states with $n=-1$ or $n=2$ electrons on the island become important. For small gaps $\Delta \rightarrow 0$ also Eq. (12) reproduces the NNN result of Eq. (5).

Interpretation of results in terms of average energy. The asymptotic results (11) and (12) can be intuitively understood in terms of Eq. (4) by considering the average electron energy $\langle\xi\rangle$ of the dominating transport mechanism. In Fig. 3 we schematically present the transport mechanisms for potentials $\phi$ such that $\left|u_{0}(\phi)\right|<\Delta$. The two processes corresponding to the rate $\Gamma_{r}^{1 \rightarrow 0}$, Eq. (7), are shown. The Fermi level of the lead is chosen to be zero. The average energy of the electrons involved in process (A) is then $\left\langle\xi_{A}\right\rangle \approx u_{0}(\phi)+\Delta>0$. On the other hand, process (B) involves the breaking of a Cooper pair: one electron tunnels to the lead while the other remains as a quasiparticle on the island. Hence, the average energy of the outgoing electrons is given by $\left\langle\xi_{B}\right\rangle \approx u_{0}(\phi)-\Delta<0$.

As can be seen from Fig. 3, the two processes are not equally likely to occur due to the different occupation numbers in the island and the lead. We can read off a lowtemperature estimate for the probabilities from Fig. 3: $p_{A}$ $\propto \exp [-\Delta / T]$ and $p_{B} \propto \exp \left[-\left(\Delta-u_{0}\right) / T\right]$. Thus, relation (4) together with the condition $p_{A}+p_{B}=1$ leads to the estimate 
$S=-\frac{1}{e T}\left(p_{a}\left\langle\xi_{A}\right\rangle+p_{B}\left\langle\xi_{B}\right\rangle\right)=-\frac{1}{e T}\left(u_{0}(\phi)-\Delta \tanh \left[\frac{u_{0}(\phi)}{2 T}\right]\right)$.

This corresponds precisely to the result (11) in the lowtemperature limit $\Delta / T \gg 1$ where $\widetilde{\Delta}(T) \rightarrow \Delta$.

If the potential $\phi$ increases further such that $u_{0}(\phi)<-\Delta$, process (A) clearly dominates as $p_{A} \gg p_{B}$ and one can neglect process (B) entirely. However, the tunneling of low-lying quasiparticle excitations is also strongly suppressed. By including the energy dependence of the density of states in the superconductor we find an estimate for the average energy $\left\langle\xi_{A}\right\rangle$

$$
\left\langle\xi_{A}\right\rangle \approx u_{0}(\phi)+\frac{\left\langle E_{(1)}\right\rangle}{\left\langle E_{(0)}\right\rangle} \quad \text { with }
$$

$$
\left\langle E_{(k)}\right\rangle \equiv \int_{\Delta}^{-u_{0}(\phi)} d E E^{k} \frac{E}{\sqrt{E^{2}-\Delta^{2}}} .
$$

Including this estimate into relation (4) directly yields the result (12).

In conclusion, we have developed a theory for the thermopower $S$ of a NSN SET with $\Delta<E_{c}$. The numerically exact results are presented in Fig. 2 and the asymptotic lowtemperature behavior is given in Eqs. (11) and (12). We showed that these results can be understood on the basis of the average-energy interpretation Eq. (4). We mention that the current-voltage characteristics of a NSN SET in the relevant range of the parameters discussed in this work have already been studied in experiments. ${ }^{16,17}$ It should be well within the reach of present-day nanotechnology to experimentally detect the sensitive dependence of the thermopower on the gap size summarized in Fig. 2.

The authors would like to thank R. Fazio, R. Scheibner, and Ch. Strunk for helpful discussions. Financial support from the DFG under Contracts No. Ri 681/5-1 and SFB 631 is gratefully acknowledged. J.S. is also supported by the DFG.
*Electronic address: marko.turek@physik.uni-regensburg.de

${ }^{1}$ D. V. Averin and K. K. Likharev, in Mesoscopic Phenomena in Solids, edited by B. Altshuler, P. A. Lee, and R. A. Webb (Elsevier, Amsterdam, 1991).

${ }^{2}$ C. W. J. Beenakker and A. A. M. Staring, Phys. Rev. B 46, 9667 (1992).

${ }^{3}$ A. A. M. Staring et al., Europhys. Lett. 22, 57 (1993).

${ }^{4}$ A. S. Dzurak et al., Phys. Rev. B 55, R10197 (1997).

${ }^{5}$ M. Turek and K. A. Matveev, Phys. Rev. B 65, 115332 (2002).

${ }^{6}$ S. Möller, H. Buhmann, S. F. Godijn, and L. W. Molenkamp, Phys. Rev. Lett. 81, 5197 (1998).

${ }^{7}$ A. V. Andreev and K. A. Matveev, Phys. Rev. Lett. 86, 280 (2001).

${ }^{8}$ K. A. Matveev and A. V. Andreev, Phys. Rev. B 66, 045301 (2002).

${ }^{9}$ D. Boese and R. Fazio, Europhys. Lett. 56, 576 (2001).

${ }^{10} \mathrm{R}$. Scheibner et al., cond-mat/0410671 (unpublished).
${ }^{11}$ J. Koch, F. von Oppen, Y. Oreg, and E. Sela, Phys. Rev. B 70, 195107 (2004).

${ }^{12}$ G. Schön, J. Siewert, and A. D. Zaikin, Physica B 203, 340 (1994).

${ }^{13}$ D. V. Averin and Yu. V. Nazarov, Phys. Rev. Lett. 69, 1993 (1992); M. T. Tuominen, J. M. Hergenrother, T. S. Tighe, M. Tinkham, ibid. 69, 1997 (1992).

${ }^{14}$ A. A. Abrikosov, Fundamentals of the Theory of Metals (NorthHolland, Amsterdam, 1988).

${ }^{15}$ K. A. Matveev, in Statistical and Dynamical Aspects of Mesoscopic Systems, edited by D. Reguera, G. Platero, L. L. Bonilla, and J. M. Rubi, Proceedings of the XVI Sitges Conference on Statistical Mechanics, Sitges, Barcelona, Spain, 1999, p. 3-15.

${ }^{16}$ T. M. Eiles, J. M. Martinis, and M. H. Devoret, Phys. Rev. Lett. 70, 1862 (1993).

${ }^{17}$ J. M. Hergenrother, M. T. Tuominen, and M. Tinkham, Phys. Rev. Lett. 72, 1742 (1994). 